\title{
G scomem \\ Drug Repurposing of $\beta$-blocker Propranolol in Osteosarcoma: Preclinical Antitumor Efficacy Alone or in Combination with Chemotherapy.
}

\section{Luisina M. Solernó}

Molecular Oncology Lab, Science and Technology Department, National University of Quilmes, Buenos Aires, Argentina

\section{Natasha T. Sobol}

Molecular Oncology Lab, Science and Technology Department, National University of Quilmes, Buenos Aires, Argentina

\section{Carla S. Capobianco}

Molecular Oncology Lab, Science and Technology Department, National University of Quilmes, Buenos Aires, Argentina

\section{Liliana Vásquez}

Precision Medicine Research Center, School of Medicine, University of San Martín de Porres, Lima, Perú.

\section{Giselle V. Ripoll}

Molecular Oncology Lab, Science and Technology Department, National University of Quilmes, Buenos Aires, Argentina. National Council of Scientific and Technical Research (CONICET), Buenos Aires,

Argentina

\section{Daniel F. Alonso}

Molecular Oncology Lab, Science and Technology Department, National University of Quilmes, Buenos Aires, Argentina. National Council of Scientific and Technical Research (CONICET), Buenos Aires,

Argentina

Juan Garona ( $\nabla$ juan.garona@unq.edu.ar)

Molecular Oncology Lab, Science and Technology Department, National University of Quilmes, Buenos Aires, Argentina. https://orcid.org/0000-0002-0389-5546

\section{Research}

Keywords: Propranolol, $\beta$-blocker, osteosarcoma, drug repurposing, metronomics, coadjuvant therapy

Posted Date: July 22nd, 2020

DOl: https://doi.org/10.21203/rs.3.rs-44912/v1 
License: (c) (i) This work is licensed under a Creative Commons Attribution 4.0 International License. Read Full License 


\section{Abstract}

Background: Osteosarcoma is still associated with poor response to therapy and alarmingly elevated mortality rates, especially in developing countries, highlighting the urgent need of novel therapeutic strategies. Given that $\beta$-adrenergic receptor ( $\beta$-AR) signaling regulates many cellular processes involved in the initiation and progression of cancer, multiple efforts have been made to repurpose non-selective $\beta$ blocker propranolol in oncology. Considering the unsatisfied clinical needs of osteosarcoma, we evaluated the in vitro and in vivo antitumoral activity of propranolol as a monotherapy or in combination with metronomic chemotherapy, in $\beta$-AR-expressing human osteosarcoma cells.

Methods: In vitro anti-osteosarcoma effects of propranolol were assessed on high-density cell growth, chemotaxis, colony-forming ability and three-dimensional spheroids. Cell cycle phase distribution and apoptosis were evaluated using flow cytometry analysis, and quantification of hypodiploid cell population and TUNEL labelling, respectively. Propranolol addition to chemotherapy was evaluated in vitro and in human osteosarcoma xenografts generated in athymic mice.

Results: Propranolol significantly impaired MG-63 and U-2 OS cellular growth in a concentration range of $10-100 \mu \mathrm{M}\left(\mathrm{IC}_{50} \approx 45 \mu \mathrm{M}\right)$ and was capable of blocking in vitro protumoral effects triggered by catecholamines. Cytostatic activity of propranolol was associated to reduced mitosis and $G_{0} / G_{1}$ cell cycle, but not to apoptosis induction. Moreover, $\beta$-blocker drastically reduced colony formation, spheroid progression and migration of osteosarcoma cells. Synergistic effects were observed in vitro after combining propranolol with chemotherapeutic agents methotrexate or cisplatin. In vivo, treatment with propranolol (10 mg/kg i.p.), alone and especially in addition to non-interrupted low-dose cisplatin (2 $\mathrm{mg} / \mathrm{kg}$ i.p.), markedly abrogated xenograft progression, reducing tumor growth rates by 25 or $70 \%$, respectively. After histological analysis, propranolol and cisplatin combined therapy resulted in low tumor mitotic index and increased tumor necrosis.

Conclusions: Considering its robust antitumoral effects, $\beta$-AR antagonization using $\beta$-blocker propranolol seems to be an achievable and cost-effective therapeutic approach to modulate osteosarcoma aggressiveness, in addition to chemotherapy. Propranolol could be proposed as a co-adjuvant agent for the management of osteosarcoma, especially in low- and middle-income countries where access to treatment resources are limited. Further preclinical and clinical studies of propranolol repurposing in osteosarcoma are warranted.

\section{Background}

Osteosarcoma (OSA) is the most prevalent primary bone tumor, affecting mostly pediatric and juvenile age-group patients [1]. Despite global 5-year overall survival in OSA patients is near to $70 \%$, there is a welldocumented disparity regarding patient survival between high-income countries (HIC) and low-middle income countries (LMICs) [2]. In addition to the surgical removal of primary and secondary lesions, management of OSA involves adjuvant administration of chemotherapy, including cisplatin and 
methotrexate [3]. However, chemotherapy effectiveness in OSA patients is restricted by the development of drug resistance by tumor cells, lack of selectivity and high toxicity [4,5]. Taking this into account, there is an urgent need to develop novel, cost-effective and selective therapeutic alternatives for the treatment of aggressive OSA, putting emphasis on relevant biological events that rule disease progression.

Drug repurposing in oncology is defined as the implementation of already-approved drugs for other medical applications to treat cancer. In comparison to de novo drug discovery, both development risks and costs, as well as safety-related failure, are reduced with this approach because such drugs have wellknown pharmacokinetic/pharmacodynamics profiles, and pharmacovigilance for adverse effects is largely accessible [6, 7]. Moreover, seeking for enhanced therapeutic benefits, repurposed drugs are often combined with metronomic chemotherapy, consisting in frequent administrations of low-dose chemotherapy, with no sustained drug-free breaks [8]. This treatment strategy is called "metronomics", and stands as an interesting therapeutic approach to especially implement in LMICs as its reduced costs and fewer infrastructure needs can be easily adapted in resource-limited countries [9].

Propranolol (PPN) is a non-selective $\beta$-adrenergic receptor ( $\beta$-AR) antagonist that was originally used for the treatment of hypertension and other cardiovascular conditions [10]. Over the last years, PPN has demonstrated potent anticancer effects in a wide variety of oncological indications. After initial clinical retrospective observations and subsequent clinical trials, this $\beta$-blocker was first repurposed for the treatment of infantile hemangioma, a common vascular tumor of infancy [11]. Preclinical studies showed that $\beta$-AR type 1 and 2 blockade using PPN results in cell cycle arrest, apoptosis and proliferation reduction of breast, melanoma and liver tumor cells, among others, mainly by disrupting mitogenic signaling cascades involving protein kinase $B(P K B, A K T)$, mitogen activated protein kinase (MAPK) and cyclic adenosine monophosphate (CAMP) [12-14]. Moreover, PPN demonstrated to reverse protumoral effects mediated by stress hormones in chronic or surgical stress models, reducing angiogenesis and metastasis, and increasing immune function $[10,15]$. Taking all these data into account, assessment of PPN anticancer properties has recently reached clinical stages for angiosarcoma, ovarian cancer and melanoma, mainly as a surgical adjuvant or in combination with chemotherapy or biologic targeted therapy [16-18].

Despite sympathetic nervous system (SNS) activation and signaling pathways triggered by catecholamines such as epinephrine and norepinephrine are deeply involved in cancer initiation and progression $[19,20]$, little is known about the specific role of catecholamines or $\beta$-AR signaling in OSA. As recently reported, expression of $\beta$-ARs and several catecholamines, including norepinephrine, was significantly higher in OSA in comparison to healthy bone [21]. In addition, all subtypes of $\beta$-AR were found to be expressed in a wide collection of human and canine OSA or other bone sarcoma models [2224], making the exploration of non-selective $\beta$-blocker PPN in OSA an appealing niche for further studies. Considering the unsatisfied clinical needs of OSA and the multiple reported benefits of PPN as a broad antitumor agent, the aim of the present study was to evaluate the in vitro/in vivo antitumoral activity of PPN in OSA, as a monotherapy or in combination with metronomic chemotherapy. 


\section{Methods}

Drugs and compounds. DL-PPN hydrochloride lyophilized powder (Sigma-Aldrich, Missouri, US) was first solubilized using citrate buffer $(\mathrm{pH} 3)$ and further diluted with phosphate buffer saline (PBS) to reach working concentrations. Cisplatin (CDDP) and methotrexate (MTX) were purchased from Fada (Buenos Aires, Argentina) and Abbot laboratories (Illinois, US), respectively. Catecholamines epinephrine and norepinephrine were purchased from BIOL laboratories (Buenos Aires, Argentina).

Tumor cell lines. Human MG-63 (ATCC $\AA$ CRL-1427 ${ }^{\mathrm{TM}}$ ) and U-20S (ATCC $\AA$ HTB-96 ${ }^{\mathrm{TM}}$ ) OSA cell lines and glioma U-87MG cells (ATCC $\AA$ HTB-14 ${ }^{\mathrm{TM}}$ ) were grown in Dulbecco's modified Eagle's medium (DMEM) (Gibco, Massachusetts, US) plus 10\% fetal bovine serum (FBS) (Natocor, Córdoba, Argentina), 2 mM glutamine and $80 \mu \mathrm{g} / \mathrm{ml}$ gentamycin in monolayer culture, at $37^{\circ} \mathrm{C}$ in a humidified atmosphere of $5 \% \mathrm{CO}_{2}$. All cells were harvested using a trypsin/EDTA solution (Gibco, Massachusetts, US) and routinely tested por mycoplasma.

OSA cell growth and citotoxicity. Direct in vitro effects of PPN, alone or in addition to chemotherapy, or catecholamines, against rapidly growing MG-63 or U-2 OS OSA cells were assessed by crystal violet staining after a 72-h treatment (for detailed methodology see Additional file 4. Supplementary methods). Before evaluating the effects of PPN on cell cycle phase distribution or migration, lack of direct short-term cytotoxicity of PPN was confirmed using the trypan blue dye exclusion assay (for detailed methodology see Additional file 1. Supplementary methods).

Cell cycle distribution. Cell cycle phase distribution and hypodiploid population quantification in starved OSA cells following a $24 \mathrm{~h}$ PPN $(50 \mu \mathrm{M})$ treatment was evaluated by flow cytometry as previously reported [25] (for detailed methodology see Additional file 1. Supplementary methods).

Apoptosis. After a 48-h treatment with PPN $(50 \mu \mathrm{M})$, apoptotic cells in OSA cultures were detected by terminal deoxynucleotidyl transferase dUTP nick end labeling (TUNEL) and fluorescence microscopy (for detailed methodology see Additional file 1. Supplementary methods).

In vitro mitosis. PFA-fixed and DAPI-stained OSA cell cultures were analyzed for mitotic index calculation after a 48-h exposure to PPN $(50 \mu \mathrm{M})$. Mitotic figures in all four basic stages of mitosis were counted using a high-power field (HPF) of 400 -fold magnification (X400) and the percentage of mitotic cells was obtained after quantification of total cell number. Ten pictures per group were used for quantification.

Clonogenic growth. Effect of PPN $(1,10$ or $50 \mu \mathrm{M})$ on OSA colony forming ability was assessed using a 7-d clonogenic assay, as previously reported [25] (for detailed methodology see Additional file 1. Supplementary methods).

OSA spheroid growth. Impact of PPN $(50 \mu \mathrm{M})$ treatment on 3D growth was evaluated on MG-63 multicellular spheroids generated by the hanging drop method [26]. After confirming appropriate sphericity and size (> $200 \mu \mathrm{m})$, fully-formed spheroids were incubated with PPN during one week, and 
volume was periodically assessed as a direct parameter of 3D OSA growth (for detailed methodology see Additional file 1. Supplementary methods).

Cell chemotaxis. Migration was assessed in serum-starved MG-63 cell after 24-h treatment with PPN $(50 \mu \mathrm{M})$ using the Transwell $\circledast$ migration assay, as previously reported [27] (for detailed methodology see Additional file 1. Supplementary methods).

Animals. Outbred athymic female $\mathrm{N}: \mathrm{NIH}(\mathrm{S})$-nu mice aged 8 weeks with a weight of approximately $23 \mathrm{~g}$, were purchased from the School of Veterinary Sciences Animal Facility at National University of La Plata (Buenos Aires, Argentina), and, after randomization, housed at 5 mice per cage in our animal facility at the National University of Quilmes. Food and water were provided ad libitum and general health status of the animals was monitored daily.

OSA xenograft progression. Human OSA tumors were heterotopically generated after subcutaneous injection of MG-63 cells in athymic mice [28]. In vivo combination studies were performed by administering PPN $10 \mathrm{mg} / \mathrm{kg}$ i.p. in a 5-day-on, 2-day-off schedule, alone or in combination with $2 \mathrm{mg} / \mathrm{kg}$ i.p. doses of CDDP three times per week during 4 weeks, until the end of the protocol. PPN $[29,30]$ and $\operatorname{CDDP}[31,32]$ dosage were defined according to previously reported preclinical studies following a metronomic rationale. Tumor growth rates and volume, as well as total animal weight were recorded or calculated throughout the protocol. Histopathological assessment of OSA tumors involved mitotic index quantification in viable sections of H\&E-stained tumor slides and determination of adjusted tumor necrotic rate after treatment [33] (for detailed methodology see Additional file 1. Supplementary methods).

Statistics. Statistical analysis was performed using the PRISM 6 (GraphPad Software Inc., San Diego, US) or Compusyn software (Combosyn Inc., New Jersey, US). To compare differences between two experimental groups Mann Whitney or $t$ tests were used for non-parametric or normal distribution of data, respectively. In case of more than two experimental groups, ANOVA analysis with Tukey's multiple comparisons post-test was used when normal distribution of data was determined. Kruskal-Wallis analysis with Dunn's multiple comparisons post-test was used in case of non-parametric distribution of data. Differences were considered statistically significant at a level of $p<0.05$. Data correspond of at least 2 or 3 independent experiments unless stated otherwise. Data were presented as mean \pm standard deviation (SD) or standard error of mean (SEM).

\section{Results}

First, in order to evaluate in vitro OSA sensitivity to PPN, semiconfluent monolayers of rapidly growing MG-63 and U-2 OS cells were exposed to tested compound using a concentration range between $1 \mu \mathrm{M}$ and $1 \mathrm{mM}$, as determined based on previously reported preclinical studies $[13,14,34]$. After a $72-h$ exposure to PPN, we observed a clear dose-dependent cell growth inhibition in both tested cell lines obtaining $\mathrm{IC}_{50}$ values of 45.6 and $47 \mu \mathrm{M}$, respectively (Fig. 1a). At high concentrations however, MG-63 
showed an increased sensitivity to PPN, obtaining more than a $95 \%$ growth reduction at $100 \mu \mathrm{M}$ or higher. As expected, human glioma U-87MG cells, which do not express $\beta 1$-AR or $\beta 2$-AR [35], were exposed to $50 \mu \mathrm{M}$ PPN $\left(\approx \mathrm{IC}_{50}\right.$ in responsive cells) and showed a complete lack of cytostatic effect by the compound (Fig. 1b). Both OSA cell lines are positive for $\beta 1$-AR and $\beta 2$-AR expression, and are responsive to catecholamine agonistic stimulation [22, 23]. After 72-h stimulation, catecholamines epinephrine and norepinephrine increased OSA cell proliferation, showing a maximum protumoral effect at $10 \mathrm{nM}$ (data not shown). In this context, coincubation with PPN using a low $10 \mu \mathrm{M}$ concentration was capable of completely blocking the protumoral activity of catecholamines (Fig. 1c).

A significant arrest in the $G_{0} / G_{1}$ cell cycle phase was observed following a 24-h treatment using a $50 \mu \mathrm{M}$ concentration of PPN as evaluated by flow cytometry studies. This arrest was accompanied by a decline of cell populations in both $S$ and $G_{2} / M$ phases (Fig. $1 \mathrm{~d}$ ). To discard any short-term direct cytotoxicity of PPN on such treatment schedule, semiconfluent MG-63 cultures exposed to PPN were assessed by the trypan blue dye exclusion assay, showing that PPN at $\approx \mathrm{IC}_{20}$ and $\mathrm{IC}_{50}$ concentrations had no effect on cell viability (data not shown). Interference of cell cycle progression after $\beta$-blockage was further confirmed by in vitro mitotic index calculation, in which PPN caused a dramatic $80 \%$ reduction in the $\%$ of mitotic cells in OSA cultures (Fig. 1e and f). Despite the large amount of preclinical/clinical data reporting the pro-apoptotic effects of PPN on malignant cells [12, 14], PPN at a concentration $\approx \mathrm{IC}_{50}$ wasn't capable of inducing apoptosis in OSA cells, as evaluated by quantification of hypodiploid sub- $\mathrm{G}_{0} / \mathrm{G}_{1}$ cell population and TUNEL labeling (Fig. $1 \mathrm{~g}$ and $\mathrm{h}$ ).

Early metastatic spread and recurrences after tumor removal are strongly related to poor prognosis in OSA [36]. Considering that tumor cell migration, colony establishment and microtumor outgrowth are all hallmarks of the early stages of metastatic progression, we tested the effect of $\beta$-blocker PPN on the establishment and progression of 2D tumor colonies, 3D spheroid growth and OSA cell chemotaxis. Clonogenic growth of OSA cells was severely suppressed after 7 days of incubation with 1, 10 and $50 \mu \mathrm{M}$ of PPN, reducing colony formation capacity by 31,46 and $100 \%$, respectively, in comparison to saline vehicle-treated cells (Fig. 2a). In this experimental setting, an IC 50 $_{0}$ value of $19.26 \mu \mathrm{M}$ was obtained on long-term cell cultures, a concentration 2.5-fold lower than that obtained for exponentially-growing cells. As above-mentioned, anti-OSA activity was also evaluated on established multicellular spheroids during a 7-day treatment period. In contrast to control spheroids which grew until reaching a 6-fold increase in volume, growth of PPN-treated spheroids was completely blocked (Fig. 2bi, ii and iii), as observed in representative photographs or individual spheroid progression curves. Chemotaxis of MG-63 cells after an overnight exposure to PPN was also significantly impaired, causing a cell migration reduction of $48 \%$ (Fig. 2c).

Anti-metabolite MTX and DNA-damaging agent CDDP remain as cornerstones in the treatment of OSA [3]. With the aim of evaluating potential therapeutic benefits after addition of PPN to metronomic chemotherapy, we first conducted in vitro combination studies exploring the cytostatic effects of PPN plus MTX or CDDP. After calculating $\mathrm{IC}_{50}$ values for MTX and CDDP as monotherapies (Additional file 2. 
Fig. S1a and b), sub- and supra-IC $\mathrm{C}_{50}$ concentrations of PPN and cytotoxic agents were defined for combination assays. Synergy was observed in all low- and high-concentration combination scenarios after addition of PPN to MTX (Fig. 3a) or CDDP (Fig. 3b) (Specific Cl and DRI values can be found in Additional files 3 and 4. Tables S1 and S2, respectively). Only in the PPN $50 \mu \mathrm{M}$ plus MTX $10 \mathrm{nM}$ combination, addition of PPN was unable to improve MTX anti-OSA activity (Fig. 3a, right). Taking these results into consideration, in addition to previous studies reporting cooperative activity between PPN and CDDP-based chemoradiotherapy in different tumor types [37, 38], the anti-OSA activity of PPN in combination with CDDP was further evaluated in vivo on MG-63 xenografts (Fig. 4a). Treatment with PPN in addition to metronomic CDDP dramatically hindered OSA xenograft progression, reducing tumor growth rate by $70 \%$ in comparison to vehicle-treated mice and significantly improving the anti-OSA activity of PPN or CDDP alone (Fig. 4b). PPN treatment as a single agent was also capable of reducing tumor growth, causing a less profound reduction of about $20 \%$. Therapeutic benefits of PPN plus CDDP were confirmed after measuring final tumor burden, were mean tumor weight was reported as $146.7 \pm$ $38.9 \mathrm{mg}$ in control group in comparison to $53 \pm 23.8 \mathrm{mg}$ (mean \pm SD) in the PPN plus CDDP group (Fig. 4c). Furthermore, all treatments were well tolerated as no significant changes in animal body weight, food or water consumption were observed throughout the protocol (Fig. 4d). Representative OSA tumorbearing mice from different experimental groups are depicted in Fig. 4e.

Given that high mitotic counts are associated to OSA aggressiveness and poor prognosis [39], mitotic index was assessed in resected tumors obtaining values of $8.3 \pm 2.7,5 \pm 2.3,3.36 \pm 2.1$ and $2.81 \pm 2.2$ mitotic bodies/HPF for PPN, CDDP and PPN + CDDP groups, respectively (Fig. 5a and b). Despite sustained PPN + CDDP therapy was associated to the most drastic drop in mitotic counts $(67 \%$ inhibition), all tested therapies achieved significant reductions. Interestingly, despite not being reflected on its effect on OSA growth, sustained low-dose CDDP treatment was linked to an evident reduction in the proliferating status of OSA cells within the tumor, quite equivalent to combined therapy. Considering that assessment of tumor necrosis in OSA has become a relevant tool for evaluating response to therapy [40, 41], adjusted tumor necrotic rates (ATNR) were calculated for all experimental groups (Fig. 5c). Only PPN plus CDDP-based therapy was capable of significantly enhancing ATNR, from $58.32 \pm 5.04$ to $81.74 \pm$ $2.25 \%$. Besides a marked increase in ATNR, tumors belonging to PPN + CDDP-treated animals displayed a peculiar diffuse and rather centered pattern of viable tumor tissue in contrast to control, PPN or CDDP groups, where viable tumor tissue areas were found as large and scattered basophilic regions, interrupted by vast areas of necrotic tissue (Fig. 5 d).

\section{Discussion}

To the best of our knowledge this is the first study to show therapeutic benefits of non-selective $\beta$-AR antagonist PPN in combination with chemotherapeutic agents in human OSA models. Exposure of OSA cells to PPN was associated to reduced proliferation and mitosis, $G_{0} / G_{1}$ cell cycle arrest and impairment of $2 \mathrm{D} / 3 \mathrm{D}$ tumor colony formation and growth. With an $\mathrm{IC}_{50} \approx 50 \mu \mathrm{M}$, sensitivity of OSA cells to PPN appears equivalent or even higher compared to other aggressive pediatric solid tumor cell lines with 
reported $I C_{50}$ ranging from 114 to $>200 \mu \mathrm{M}[14,34]$. Despite no significant proapoptotic activity of PPN on OSA cells was observed at $50 \mu \mathrm{M}$, it is worth noting that apoptosis induction in other preclinical studies using PPN on non-OSA tumor models was achieved using concentrations in the range of 100$300 \mu \mathrm{M}[12,14,34]$. Exploring such concentrations goes beyond the scope of the present work given that, according to early pharmacokinetics reports, clinically relevant concentrations of PPN are in the low $\mu M$ range $[42,43]$. PPN cytostatic activity in $\beta$-AR expressing malignant cells was previously associated to the down-regulation of the AKT/mammalian target of rapamycin (mTOR) and the MEK/ERK1/2 MAPK signaling pathways [12-14]. Interestingly, with the aim of shedding some light into the largely unknown molecular landscape of OSA, genomic and transcriptomic studies have recently identified frequently mutated genes and copy number alterations in OSA samples [44, 45]. Besides confirming TP53, RB1 and MYC as driver genes, it was reported that PI3K/AKT/mTOR and MAPK pathways are heavily altered in OSA and are central players in disease initiation and progression. According to these data, PPN could be capable of blocking key protumoral signaling pathways in OSA biology. Thus, further studies exploring specific molecular mechanisms associated to PPN anti-OSA effects are mandatory.

After addition of PPN to CDDP or MTX, synergistic anti-OSA effects were observed in all in vitro combination studies. Increased antitumor activity as a result of combining PPN with cytotoxic agents was previously reported in non-OSA preclinical and clinical studies, in which addition of repurposed drug to paclitaxel-, 5-FU- or even CDDP-based chemotherapy increased antiangiogenic and antiproliferative effects of monotherapies, as well as decreased distant metastases in patients [30,37,38]. Moreover, it was recently reported that $\beta$-blockade in different bone sarcoma models, increases sensitivity to radiotherapy, especially in canine OSA [24]. In our in vivo protocol sustained treatment with PPN, alone and especially in addition to CDDP, caused a marked decrease in OSA tumor progression, showing that PPN may complement chemotherapy, increasing its effectiveness without overt toxicity. After histological assessment of OSA tumors, we observed a significant increase in combined treatment-associated necrosis as well as a profound reduction in mitotic indices. These findings bear high translational relevance given that necrosis rate in response to therapy and mitotic index determination are two histopathological parameters routinely used in the clinical management of OSA, linked to disease aggressiveness and prognosis $[33,39]$. Besides complementing chemotherapy, $\beta$-blockade using PPN could also show benefit during resection of primary or secondary OSA tumors in order to antagonize several deleterious mechanisms triggered by surgery, including inflammation and release of catecholamines, activation of proangiogenic and protumoral signaling pathways, tumor cell shedding, among others [46].

In our study, PPN anti-OSA activity was related to direct cytostatic effects and cell cycle arrest, decreasing survival and mitosis in $\beta$-AR expressing tumor tissue. However, SNS activation and $\beta$-adrenergic signaling can regulate a wide range of cancer-associated molecular pathways via both direct effects on $\beta$-AR expressing transformed cells and regulation of other $\beta$-receptor-bearing cells populating the complex tumor stroma, such as vascular or immune cells $[47,48]$. In spite of using T cell-deficient nude mice for our in vivo studies, this animal model still has a robust innate immune response involving dendritic cells, 
tumor-associated macrophages and natural killer cells, which are known to play a key role against OSA [49]. Taking these concepts into account, other microenvironmental mechanisms associated to pleiotropic $\beta$-blockade, such as angiogenesis inhibition or increased immune function, could be playing a part in the overall PPN anti-OSA activity and should not be discarded. For instance, PPN was shown to be highly effective and safe in the management of angiogenic diseases such as infantile hemangioma, halting the growth and ulceration of these vascular tumors $[11,50]$.

Our study has several limitations worth noting. It is widely recognized that in vivo models should recapitulate the tumor stroma as closer to the clinical setting as possible [51]. Given that PPN targets both tumor and its microenvironment, the influence of OSA tumor stroma should not be neglected, and the use of orthotopic paratibial or intraosseous tumor models should be considered for future studies. Despite showing that $\beta$-blockade by PPN affects metastasis-associated cellular events in vitro, such as 2D/3D colony growth or tumor cell chemotaxis, effects of PPN administration on in vivo metastatic spread and growth, especially to lungs, should be evaluated in order to fully characterize compound's therapeutic potential in OSA.

OSA is fatal for about one-third of the patients worldwide. However, prognosis is significantly poorer for LMICs [9]. Higher mortality rates are mainly due to economic inequalities and inefficient health-care systems, resulting in diagnosis at advanced stages and poor access to treatment resources, including surgery, radiotherapy and high-cost novel cancer drugs [2]. As previously introduced, metronomics combines drug repurposing with metronomic chemotherapy and could act as a substitute for standard treatments when unavailable or undoable [9]. PPN is a low-cost widely-available FDA-approved drug and its repositioned use in oncology is gaining strength as high quality preclinical and clinical evidence accumulates. On the other hand, in the past years metronomic chemotherapy has emerged as a promising multi-targeted therapeutic approach, showing clinical benefits in aggressive pediatric tumors with low associated-toxicities and used in outpatient settings [8, 52-55]. Therefore, due to its costeffectiveness, good tolerability and accessibility, the combination of metronomic chemotherapy in addition to repurposed PPN could represent an interesting alternative strategy to manage OSA, especially in developing countries.

\section{Conclusions}

Survival rates of OSA patients have remained practically unaltered in the last 30 years, highlighting the need of intensifying research efforts and drug development programs to tackle this disease. $\beta-A R$ antagonization seems an achievable and interesting therapeutic approach to inhibit OSA progression (Fig. 6). Integration of novel therapeutic approaches such as the use of repurposed PPN into current OSA management is both promising and challenging. We believe that this study could help lay the groundwork for translating the use of PPN as a novel adjuvant in OSA.

\section{Declarations}




\section{Ethics approval.}

All animal protocols have been carried out in accordance with the Guide for the Care and Use of Laboratory Animals as adopted by the U.S. National Institutes of Health (NIH Publications No. 8023, revised 1978) and were approved by our institutional Animal Care Committee UNQUI-CICUAL (Resolution CD CyT N075/14); Protocol code: 011-15. Results from animal studies were reported following ARRIVE guidelines.

\section{Consent for publication.}

Not applicable

\section{Availability of data and materials.}

The datasets used and/or analyzed during the current study are available from the corresponding author on reasonable request.

\section{Competing interests.}

All authors have no conflict of interests to declare.

\section{Funding.}

This work was supported by the National Agency for the Promotion of Science and Technology (ANPCYT, Argentina, grant No PICT2017/2056 to DFA, GVR and JG), the National Institute of Cancer (grant No INC2018/2020 to DFA, GVR and JG), and the National University of Quilmes Research Program grant (No PUNQ1297/19 to Dr. Daniel E. Gomez and Dr. Mariano R. Gabri).

\section{Authors' contributions.}

LMS: Formal analysis; Data curation; Investigation; Methodology; Writing - original draft. NTS: Formal analysis; Investigation; Methodology; Validation. CSC: Methodology; Visualization. LV: Writing - review \& editing. GVR: Formal analysis; Data curation. DFA: Conceptualization; Funding acquisition; Project administration; Resources; Software; Writing - review \& editing. JG: Conceptualization; Formal analysis; Funding acquisition; Investigation; Project administration; Supervision; Validation; Visualization; Writing original draft; Writing - review \& editing.

\section{Acknowledgements.}

GVR, DFA and JG are members of CONICET. NTS and CSC are doctoral and postdoctoral research fellows from CONICET, respectively. LMS is an undergraduate research fellow from the National Cancer Institute (Argentina). We would like to acknowledge the participation of Dr. Berenice Freile, M.D., and Dr. Alejandra Scursoni, M.D., especially for their involvement in histological processing and analysis. MG-63 and U-20S cell lines were kindly provided by Dr. Ignacio E. León (Laboratory of Metal based Drugs, National 
University of La Plata, Buenos Aires, Argentina) and Dr. Vanesa Gottifredi (Cell Cycle and Genomic Stability Lab, Leloir Institute Foundation, Buenos Aires, Argentina), respectively.

\section{References}

1. Meazza, C. and P. Scanagatta, Metastatic osteosarcoma: a challenging multidisciplinary treatment. Expert Rev Anticancer Ther, 2016. 16(5): p. 543-56.

2. Moreno, F., W. Cacciavillano, M. Cipolla, M. Coirini, P. Streitenberger, J. Lopez Marti, et al., Childhood osteosarcoma: Incidence and survival in Argentina. Report from the National Pediatric Cancer Registry, ROHA Network 2000-2013. Pediatr Blood Cancer, 2017. 64(10).

3. Durfee, R.A., M. Mohammed, and H.H. Luu, Review of Osteosarcoma and Current Management. Rheumatol Ther, 2016. 3(2): p. 221-243.

4. Holmboe, L., A.M. Andersen, L. Morkrid, L. Slordal, and K.S. Hall, High dose methotrexate chemotherapy: pharmacokinetics, folate and toxicity in osteosarcoma patients. Br J Clin Pharmacol, 2012. 73(1): p. 106-14.

5. Barabas, K., R. Milner, D. Lurie, and C. Adin, Cisplatin: a review of toxicities and therapeutic applications. Vet Comp Oncol, 2008. 6(1): p. 1-18.

6. Bertolini, F., V.P. Sukhatme, and G. Bouche, Drug repurposing in oncology-patient and health systems opportunities. Nat Rev Clin Oncol, 2015. 12(12): p. 732-42.

7. Pantziarka, P., C. Verbaanderd, I. Huys, G. Bouche, and L. Meheus, Repurposing drugs in oncology: From candidate selection to clinical adoption. Semin Cancer Biol, 2020.

8. Andre, N., M. Carre, and E. Pasquier, Metronomics: towards personalized chemotherapy? Nat Rev Clin Oncol, 2014. 11(7): p. 413-31.

9. Revon-Riviere, G., S. Banavali, L. Heississen, W. Gomez Garcia, B. Abdolkarimi, M. Vaithilingum, et al., Metronomic Chemotherapy for Children in Low- and Middle-Income Countries: Survey of Current Practices and Opinions of Pediatric Oncologists. J Glob Oncol, 2019. 5: p. 1-8.

10. Pantziarka, P., G. Bouche, V. Sukhatme, L. Meheus, I. Rooman, and V.P. Sukhatme, Repurposing Drugs in Oncology (ReDO)-Propranolol as an anti-cancer agent. Ecancermedicalscience, 2016. 10: p. 680.

11. Leaute-Labreze, C., P. Hoeger, J. Mazereeuw-Hautier, L. Guibaud, E. Baselga, G. Posiunas, et al., A randomized, controlled trial of oral propranolol in infantile hemangioma. N Engl J Med, 2015. 372(8): p. 735-46.

12. Montoya, A., A. Varela-Ramirez, E. Dickerson, E. Pasquier, A. Torabi, R. Aguilera, et al., The beta adrenergic receptor antagonist propranolol alters mitogenic and apoptotic signaling in late stage breast cancer. Biomed J, 2019. 42(3): p. 155-165.

13. Zhou, C., X. Chen, W. Zeng, C. Peng, G. Huang, X. Li, et al., Propranolol induced G0/G1/S phase arrest and apoptosis in melanoma cells via AKT/MAPK pathway. Oncotarget, 2016. 7(42): p. 68314-68327.

14. Wang, F., H. Liu, F. Wang, R. Xu, P. Wang, F. Tang, et al., Propranolol suppresses the proliferation and induces the apoptosis of liver cancer cells. Mol Med Rep, 2018. 17(4): p. 5213-5221. 
15. Goldfarb, Y., L. Sorski, M. Benish, B. Levi, R. Melamed, and S. Ben-Eliyahu, Improving postoperative immune status and resistance to cancer metastasis: a combined perioperative approach of immunostimulation and prevention of excessive surgical stress responses. Ann Surg, 2011. 253(4): p. 798-810.

16. Amaya, C.N., M. Perkins, A. Belmont, C. Herrera, A. Nasrazadani, A. Vargas, et al., Non-selective beta blockers inhibit angiosarcoma cell viability and increase progression free- and overall-survival in patients diagnosed with metastatic angiosarcoma. Oncoscience, 2018. 5(3-4): p. 109-119.

17. Ramondetta, L.M., W. Hu, P.H. Thaker, D.L. Urbauer, G.B. Chisholm, S.N. Westin, et al., Prospective pilot trial with combination of propranolol with chemotherapy in patients with epithelial ovarian cancer and evaluation on circulating immune cell gene expression. Gynecol Oncol, 2019. 154(3): p. 524-530.

18. De Giorgi, V., M. Grazzini, S. Benemei, N. Marchionni, E. Botteri, E. Pennacchioli, et al., Propranolol for Off-label Treatment of Patients With Melanoma: Results From a Cohort Study. JAMA Oncol, 2018. 4(2): p. e172908.

19. Lutgendorf, S.K., A.K. Sood, and M.H. Antoni, Host factors and cancer progression: biobehavioral signaling pathways and interventions. J Clin Oncol, 2010. 28(26): p. 4094-9.

20. Servick, K., War of nerves. Science, 2019. 365(6458): p. 1071-1073.

21. Bandala, C., A. Avila-Luna, M. Gomez-Lopez, E. Estrada-Villasenor, S. Montes, A. Alfaro-Rodriguez, et al., Catecholamine levels and gene expression of their receptors in tissues of adults with osteosarcoma. Arch Physiol Biochem, 2019: p. 1-7.

22. Togari, A., M. Arai, S. Mizutani, S. Mizutani, Y. Koshihara, and T. Nagatsu, Expression of mRNAs for neuropeptide receptors and beta-adrenergic receptors in human osteoblasts and human osteogenic sarcoma cells. Neurosci Lett, 1997. 233(2-3): p. 125-8.

23. Huang, H.H., T.C. Brennan, M.M. Muir, and R.S. Mason, Functional alpha1-and beta2-adrenergic receptors in human osteoblasts. J Cell Physiol, 2009. 220(1): p. 267-75.

24. Duckett, M.M., S.K. Phung, L. Nguyen, A. Khammanivong, E. Dickerson, K. Dusenbery, et al., The adrenergic receptor antagonists propranolol and carvedilol decrease bone sarcoma cell viability and sustained carvedilol reduces clonogenic survival and increases radiosensitivity in canine osteosarcoma cells. Vet Comp Oncol, 2020. 18(1): p. 128-140.

25. Garona, J., M. Pifano, U.D. Orlando, M.B. Pastrian, N.B. lannucci, H.H. Ortega, et al., The novel desmopressin analogue [V4Q5]dDAVP inhibits angiogenesis, tumour growth and metastases in vasopressin type 2 receptor-expressing breast cancer models. Int J Oncol, 2015. 46(6): p. 2335-45.

26. Zanoni, M., F. Piccinini, C. Arienti, A. Zamagni, S. Santi, R. Polico, et al., 3D tumor spheroid models for in vitro therapeutic screening: a systematic approach to enhance the biological relevance of data obtained. Sci Rep, 2016. 6: p. 19103.

27. Garona, J., N.T. Sobol, M. Pifano, V.I. Segatori, D.E. Gomez, G.V. Ripoll, et al., Preclinical Efficacy of [V4 Q5 JdDAVP, a Second Generation Vasopressin Analog, on Metastatic Spread and Tumor-Associated Angiogenesis in Colorectal Cancer. Cancer Res Treat, 2019. 51(2): p. 438-450. 
28. Garona, J., M. Pifano, M.B. Pastrian, D.E. Gomez, G.V. Ripoll, and D.F. Alonso, Addition of vasopressin synthetic analogue $[V(4) Q(5)] d D A V P$ to standard chemotherapy enhances tumour growth inhibition and impairs metastatic spread in aggressive breast tumour models. Clin Exp Metastasis, 2016. 33(6): p. 589-600.

29. Maccari, S., M. Buoncervello, A. Rampin, M. Spada, D. Macchia, L. Giordani, et al., Biphasic effects of propranolol on tumour growth in B16F10 melanoma-bearing mice. Br J Pharmacol, 2017. 174(2): $\mathrm{p}$. 139-149.

30. Pasquier, E., J. Ciccolini, M. Carre, S. Giacometti, R. Fanciullino, C. Pouchy, et al., Propranolol potentiates the anti-angiogenic effects and anti-tumor efficacy of chemotherapy agents: implication in breast cancer treatment. Oncotarget, 2011. 2(10): p. 797-809.

31. Sun, F., Y. Zhang, L. Xu, S. Li, X. Chen, L. Zhang, et al., Proteasome Inhibitor MG132 Enhances Cisplatin-Induced Apoptosis in Osteosarcoma Cells and Inhibits Tumor Growth. Oncol Res, 2018. 26(4): p. 655-664.

32. Tao, H., X. Tang, L. Jin, Y. Zhao, Y. Luo, Z. Zhang, et al., Synergistic effect of docetaxel combined with cisplatin on inhibiting human osteosarcoma in nude mice. Biochem Biophys Res Commun, 2018. 505(2): p. 372-377.

33. Kim, M.S., S.Y. Lee, W.H. Cho, W.S. Song, J.S. Koh, J.A. Lee, et al., Tumor necrosis rate adjusted by tumor volume change is a better predictor of survival of localized osteosarcoma patients. Ann Surg Oncol, 2008. 15(3): p. 906-14.

34. Wolter, J.K., N.E. Wolter, A. Blanch, T. Partridge, L. Cheng, D.A. Morgenstern, et al., Anti-tumor activity of the beta-adrenergic receptor antagonist propranolol in neuroblastoma. Oncotarget, 2014. 5(1): p. 161-72.

35. He, J.J., W.H. Zhang, S.L. Liu, Y.F. Chen, C.X. Liao, Q.Q. Shen, et al., Activation of beta-adrenergic receptor promotes cellular proliferation in human glioblastoma. Oncol Lett, 2017. 14(3): p. 38463852.

36. Spraker-Perlman, H.L., D.A. Barkauskas, M.D. Krailo, P.A. Meyers, C.L. Schwartz, J. Doski, et al., Factors influencing survival after recurrence in osteosarcoma: A report from the Children's Oncology Group. Pediatr Blood Cancer, 2019. 66(1): p. e27444.

37. Wolter, N.E., J.K. Wolter, D.J. Enepekides, and M.S. Irwin, Propranolol as a novel adjunctive treatment for head and neck squamous cell carcinoma. J Otolaryngol Head Neck Surg, 2012. 41(5): p. 334-44.

38. Chaudhary, K.R., S.X. Yan, S.P. Heilbroner, J.R. Sonett, M.B. Stoopler, C. Shu, et al., Effects of betaAdrenergic Antagonists on Chemoradiation Therapy for Locally Advanced Non-Small Cell Lung Cancer. J Clin Med, 2019. 8(5).

39. Chui, M.H., R.A. Kandel, M. Wong, A.M. Griffin, R.S. Bell, M.E. Blackstein, et al., Histopathologic Features of Prognostic Significance in High-Grade Osteosarcoma. Arch Pathol Lab Med, 2016. 140(11): p. 1231-1242.

40. Picci, P., G. Bacci, M. Campanacci, M. Gasparini, S. Pilotti, S. Cerasoli, et al., Histologic evaluation of necrosis in osteosarcoma induced by chemotherapy. Regional mapping of viable and nonviable 
tumor. Cancer, 1985. 56(7): p. 1515-21.

41. Arunachalam, H.B., R. Mishra, O. Daescu, K. Cederberg, D. Rakheja, A. Sengupta, et al., Viable and necrotic tumor assessment from whole slide images of osteosarcoma using machine-learning and deep-learning models. PLoS One, 2019. 14(4): p. e0210706.

42. Duff, H.J., D.M. Roden, L. Brorson, A.J. Wood, A.K. Dawson, R.K. Primm, et al., Electrophysiologic actions of high plasma concentrations of propranolol in human subjects. J Am Coll Cardiol, 1983. 2(6): p. 1134-40.

43. Wong, L., R.L. Nation, W.L. Chiou, and P.K. Mehta, Plasma concentrations of propranolol and 4hydroxypropranolol during chronic oral propranolol therapy. Br J Clin Pharmacol, 1979. 8(2): p. 1637.

44. Wang, H., M. Tang, L. Ou, M. Hou, T. Feng, Y.E. Huang, et al., Biological analysis of cancer specific microRNAs on function modeling in osteosarcoma. Sci Rep, 2017. 7(1): p. 5382.

45. Zhao, J., D.C. Dean, F.J. Hornicek, X. Yu, and Z. Duan, Emerging next-generation sequencing-based discoveries for targeted osteosarcoma therapy. Cancer Lett, 2020. 474: p. 158-167.

46. Hiller, J.G., N.J. Perry, G. Poulogiannis, B. Riedel, and E.K. Sloan, Perioperative events influence cancer recurrence risk after surgery. Nat Rev Clin Oncol, 2018. 15(4): p. 205-218.

47. Hanns, P., A.M. Paczulla, M. Medinger, M. Konantz, and C. Lengerke, Stress and catecholamines modulate the bone marrow microenvironment to promote tumorigenesis. Cell Stress, 2019. 3(7): p. 221-235.

48. Qiao, G., M. Chen, M.J. Bucsek, E.A. Repasky, and B.L. Hylander, Adrenergic Signaling: A Targetable Checkpoint Limiting Development of the Antitumor Immune Response. Front Immunol, 2018. 9: p. 164.

49. Wang, Z., Z. Wang, B. Li, S. Wang, T. Chen, and Z. Ye, Innate Immune Cells: A Potential and Promising Cell Population for Treating Osteosarcoma. Front Immunol, 2019. 10: p. 1114.

50. Tiwari, P., V. Pandey, A.N. Gangopadhyay, S.P. Sharma, and D.K. Gupta, Role of propranolol in ulcerated haemangioma of head and neck: a prospective comparative study. Oral Maxillofac Surg, 2016. 20(1): p. 73-7.

51. Uluckan, O., A. Segaliny, S. Botter, J.M. Santiago, and A.J. Mutsaers, Preclinical mouse models of osteosarcoma. Bonekey Rep, 2015. 4: p. 670.

52. Fousseyni, T., M. Diawara, E. Pasquier, and N. Andre, Children treated with metronomic chemotherapy in a low-income country: METRO-MALI-01. J Pediatr Hematol Oncol, 2011. 33(1): p. 31-4.

53. Verschuur, A., M.A. Heng-Maillard, P. Dory-Lautrec, R. Truillet, E. Jouve, P. Chastagner, et al., Metronomic Four-Drug Regimen Has Anti-tumor Activity in Pediatric Low-Grade Glioma; The Results of a Phase I/ Clinical Trial. Front Pharmacol, 2018. 9: p. 00950.

54. Andre, N., N. Corradini, and Y. Shaked, Metronomic Maintenance Therapy for Rhabdomyosarcoma. Trends Cancer, 2019. 5(12): p. 756-759. 
55. Berthold, F., M. Homberg, I. Proleskovskaya, P. Mazanek, M. Belogurova, A. Ernst, et al., Metronomic therapy has low toxicity and is as effective as current standard treatment for recurrent high-risk neuroblastoma. Pediatr Hematol Oncol, 2017. 34(5): p. 308-319.

\section{Figures}

a

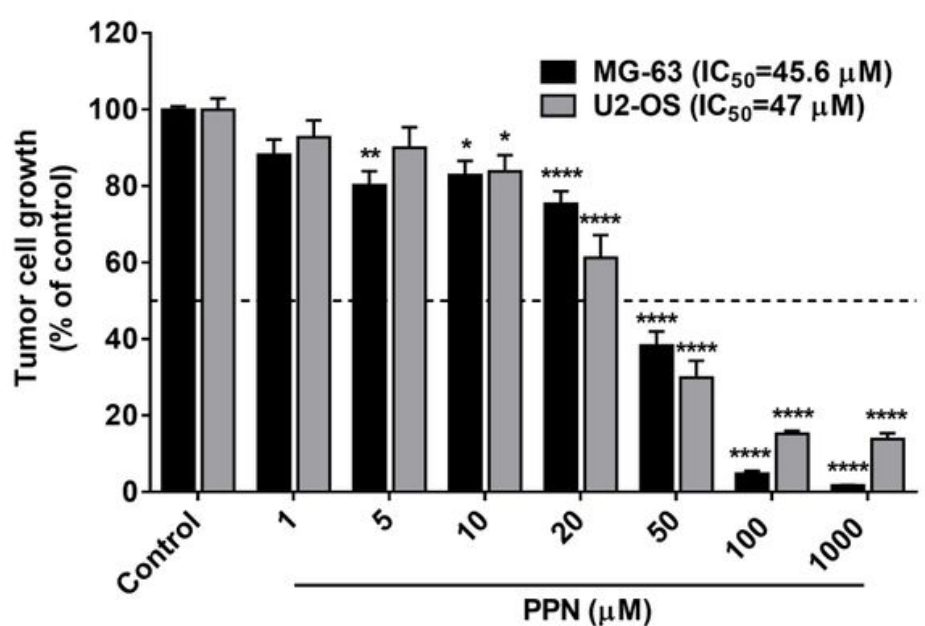

d
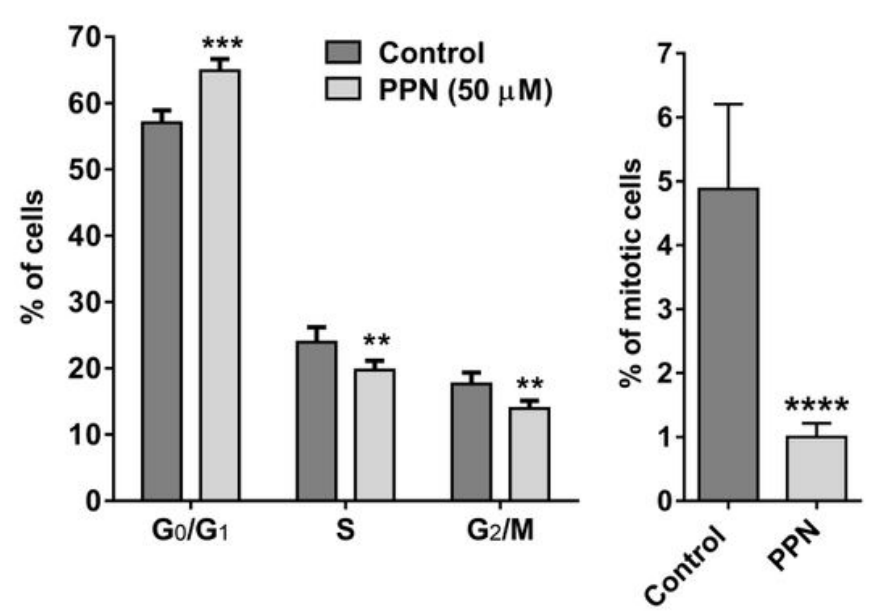

b

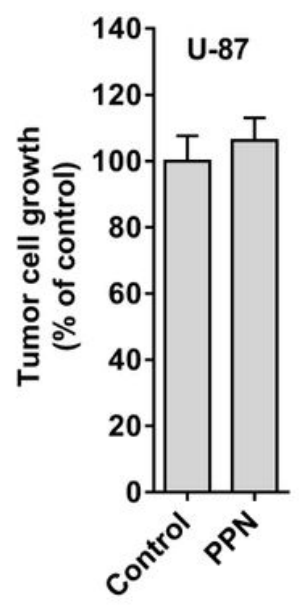

g

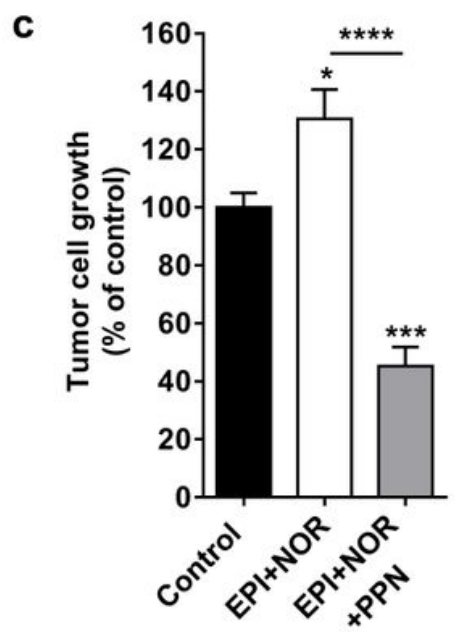

h
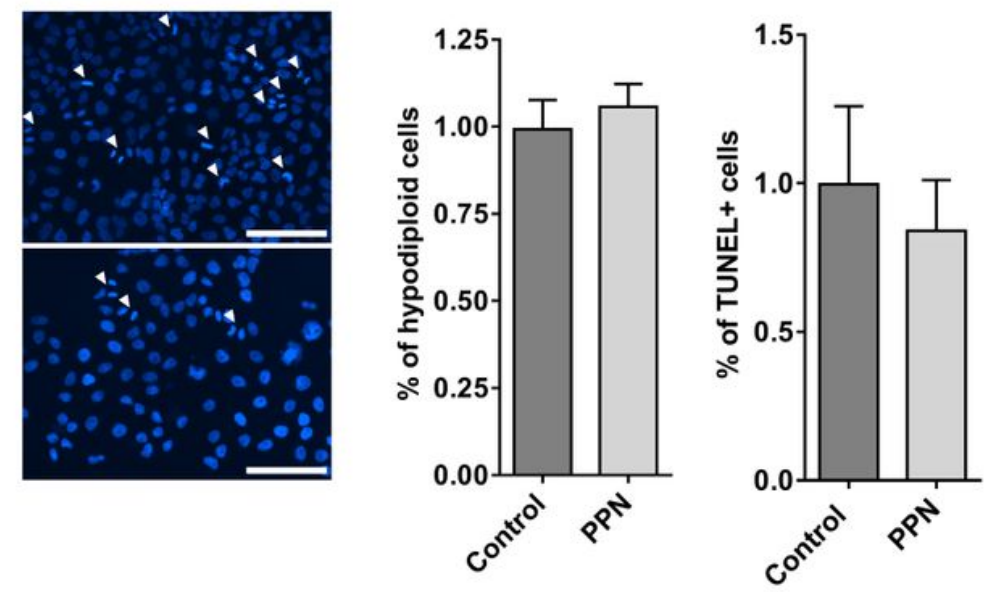

Figure 1

Direct cytostatic activity of $\beta$-blocker propranolol on osteosarcoma cells. Tumor cell growth was evaluated on log-phase growing OSA cells and measured by crystal violet staining after a 72-h exposure to tested compounds. (a) Inhibition of cell growth by PPN (1-1000 $\mu \mathrm{M})$ on MG-63 and U-2 OS cell cultures, and calculation of IC50 values. Statistical significance for PPN in both cell lines was achieved with drug concentrations $\geq$ than $10 \mu \mathrm{M}$. (b) Absence of cytostatic effect of PPN in $\beta$-AR-negative U-87MG cells. (c) Blockage of catecholamines epinephrine (EPI) and norepinephrine (NOR) promitogenic effect by coincubation with low dose PPN $(10 \mu \mathrm{M})$. (d) Cell cycle phase distribution for OSA cells treated with PPN $(50 \mu \mathrm{M})$ during $24 \mathrm{~h}$ obtained by flow cytometry analysis. (e) Calculation of in vitro mitotic index was 
performed by DAPI-stained OSA cell culture examination using fluorescence microscopy. Mitotic cells were identified after $48 \mathrm{~h}$ PPN treatment in ten HPF of X400 magnification per experimental group. (f) Representative fluorescent images of vehicle (upper) or PPN (50 $\mu \mathrm{M})$ treated (lower) OSA cultures showing different mitotic bodies (White arrows). Scale bar $=100 \mu \mathrm{m}$. (g) Cell apoptosis was evaluated as the percentage of cells in the sub-G0/G1-phase, according to their hypodiploid DNA content. (h) \% of apoptotic cells in $48 \mathrm{~h}$-treated MG-63 cell cultures were also assessed by TUNEL labelling. Data are presented as mean \pm SEM and are representative of at least two or three independent experiments. ${ }^{*} p<0.05,{ }^{*} p<0.01,{ }^{* *} p<0.001$ and ${ }^{* \star *} p<0.0001$. ANOVA followed by Tukey's test for (a) and (c), unpaired t test for (b), (d), (e) and (g), and $\chi 2$ test for (h).

a
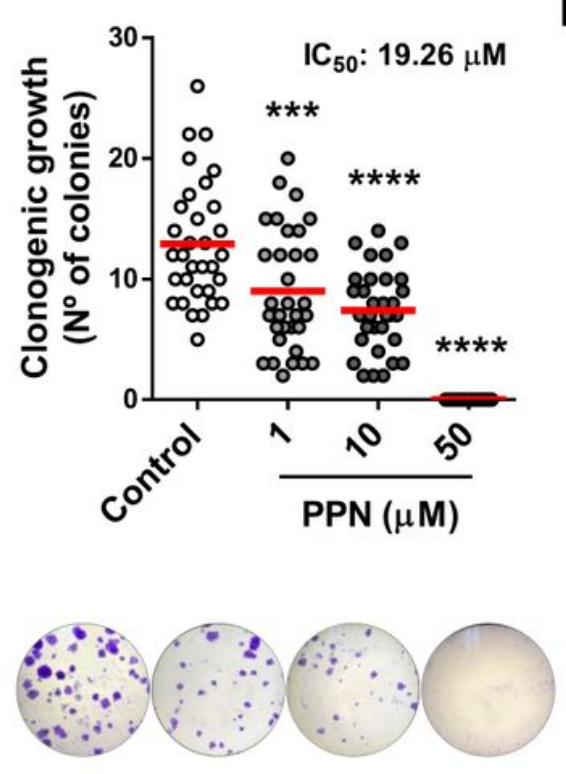

b i.

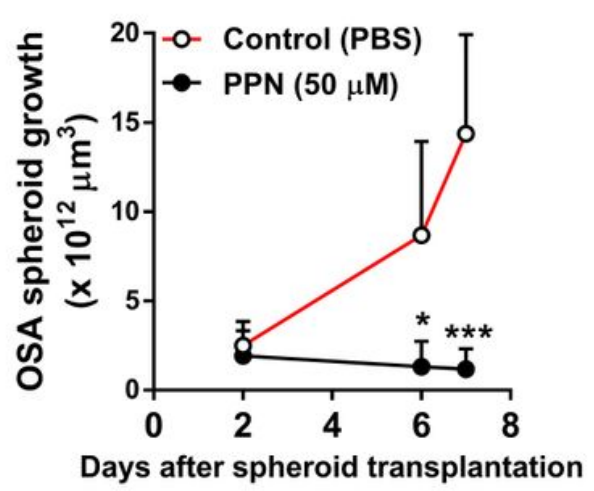

C

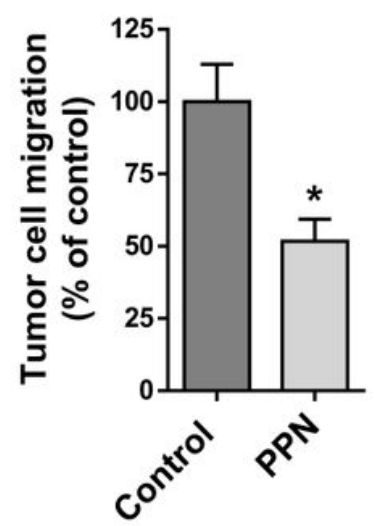

ii.

iii.

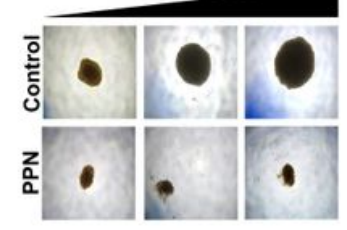

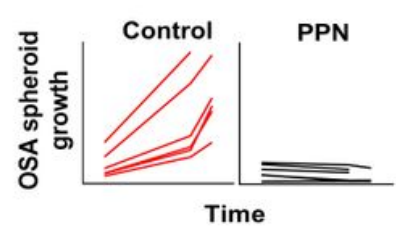

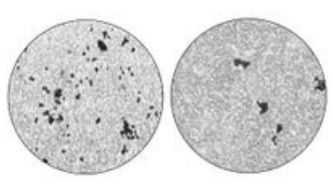

Figure 2

Propranolol abrogates colony formation, three-dimensional spheroid growth and chemotaxis in osteosarcoma. (a) Number of OSA colonies per well quadrant was quantified after a 7-d incubation with PPN (1-50 $\mu \mathrm{M})$. Representative microphotographs of MG-63 clonogenic cultures (bottom) in control and PPN 1, 10 and $50 \mu \mathrm{M}$ groups (left to right) are depicted. (b) PPN completely impairs growth of fully formed OSA spheroids. i) OSA spheroid volume was assessed as a direct parameter of spheroid growth during the following week. ii) Representative images of OSA spheroids at days 2, 6 and 7 (left to right). X40 final magnification. iii) Individual progression of vehicle- or PPN-treated 3D OSA colonies over time. (c) Inhibition of MG-63 cell chemotaxis after a 16-h treatment using PPN at $50 \mu \mathrm{M}$. Representative pictures of tumor cells on the bottom of the Transwell inserts (bottom) corresponding to control or PPN group (left and right, respectively) are shown. ${ }^{*} p<0.05,{ }^{* \star *} p<0.001$ and ${ }^{* \star * \star} p<0.0001$. ANOVA followed by Tukey's test for (a), and unpaired two-sided t test for (b) and (c). 
a

\section{Propranolol plus methotrexate}
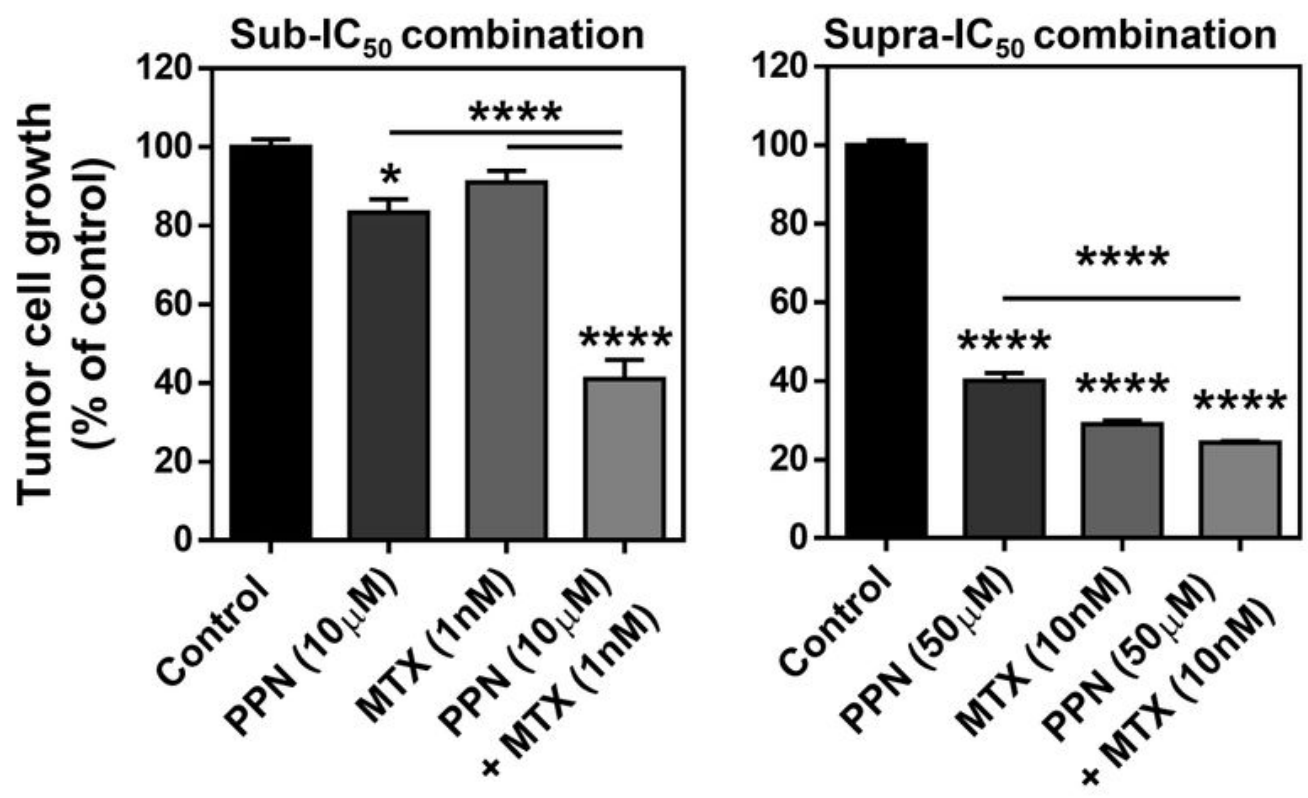

b

\section{Propranolol plus cisplatin}
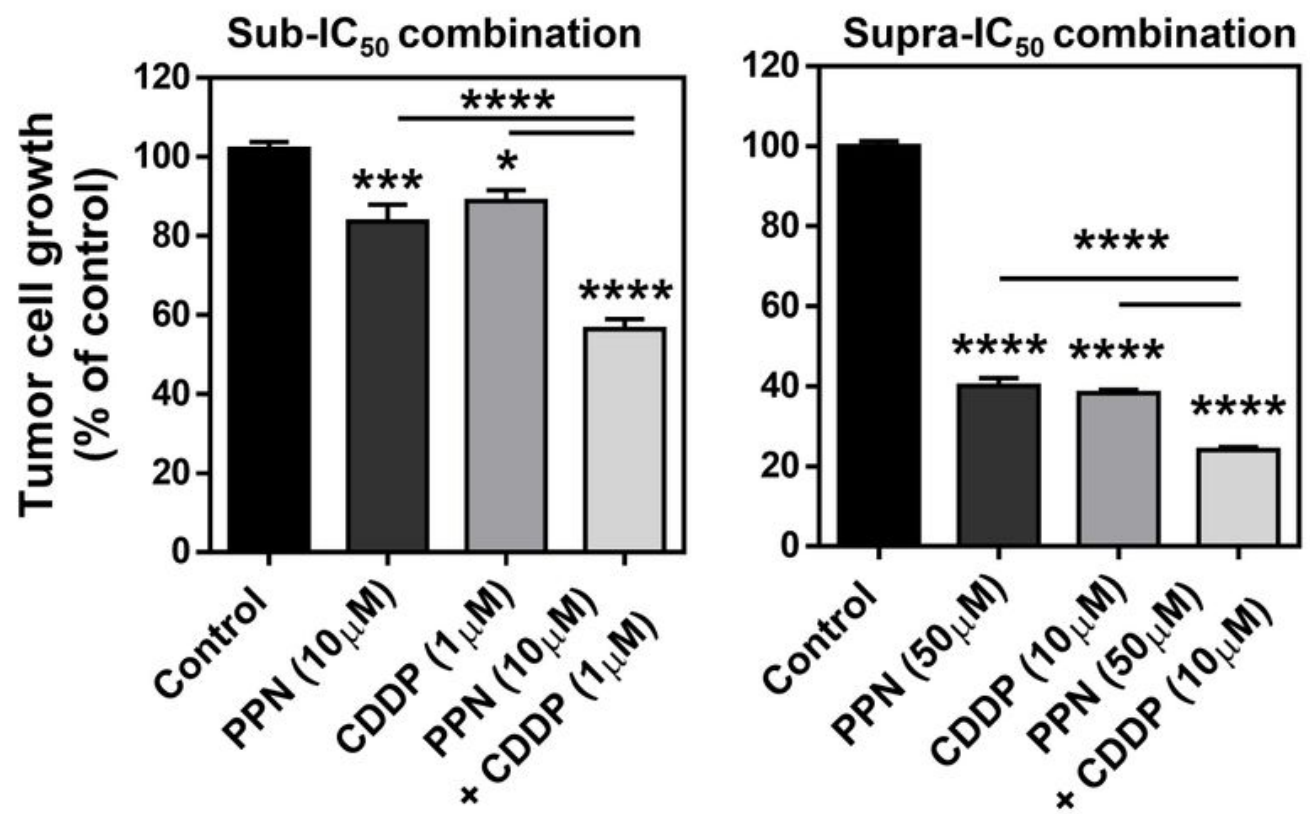

Figure 3

Synergistic anti-osteosarcoma activity of propranolol in combination with standard cytotoxic agents. Tumor cell growth was evaluated on log-phase growing MG-63 cells and measured by crystal violet staining after a 72-h drug exposure. Combinational studies were conducted after adding PPN to (a) methotrexate (MTX) or (b) cisplatin (CDDP). To test the effect on OSA cell survival, drugs were combined using sub-IC50 (left) or supra-IC50 (right) concentrations. Data are presented as mean \pm SEM and are 
representative of at least two or three independent experiments. ${ }^{*} p<0.05,{ }^{\star \star \star} p<0.001,{ }^{\star \star \star \star *} p<0.0001$. ANOVA followed by Tukey's test.
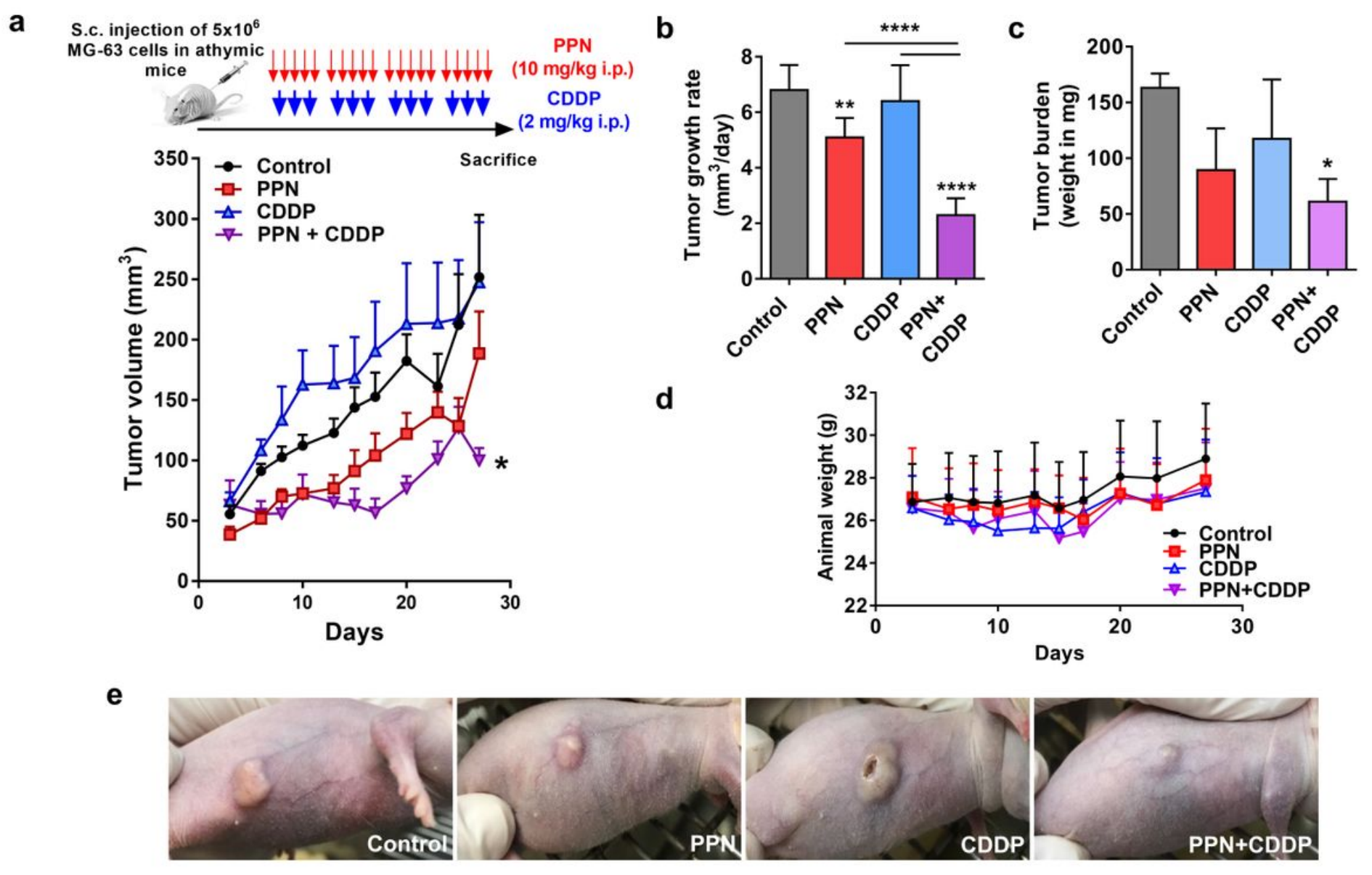

\section{Figure 4}

Sustained administration of propranolol in combination to metronomic cisplatin inhibits osteosarcoma tumor growth without overt signs of toxicity. (a) Experimental design used to evaluate in vivo effects of PPN (10 mg/kg i.p., five days per week) plus CDDP (2 mg/kg i.p., three times per week) on tumor progression of MG-63 xenografts growing in athymic mice (Top). Curves represent mean tumor volumes of mice receiving saline vehicle (control), PPN, CDDP or PPN plus CDDP over time (bottom). 5 animals per experimental group. (b) Aggressiveness of MG-63 xenografts was evaluated by calculating tumor growth rates between days 3 and 27 of protocol. (c) Final tumor burden was assessed by weighting resected OSA primary lesions after necropsy. (d) Body weight of animals bearing OSA tumors belonging to different experimental groups throughout the protocol. (e) Representative photographs of nude mice bearing xenografts belonging to different experimental groups at day 30 are shown. Data are presented as mean \pm SEM. ${ }^{\star} p<0.05,{ }^{\star *} p<0.01,{ }^{* \star \star *} p<0.0001$. ANOVA followed by Tukey's test. 
a

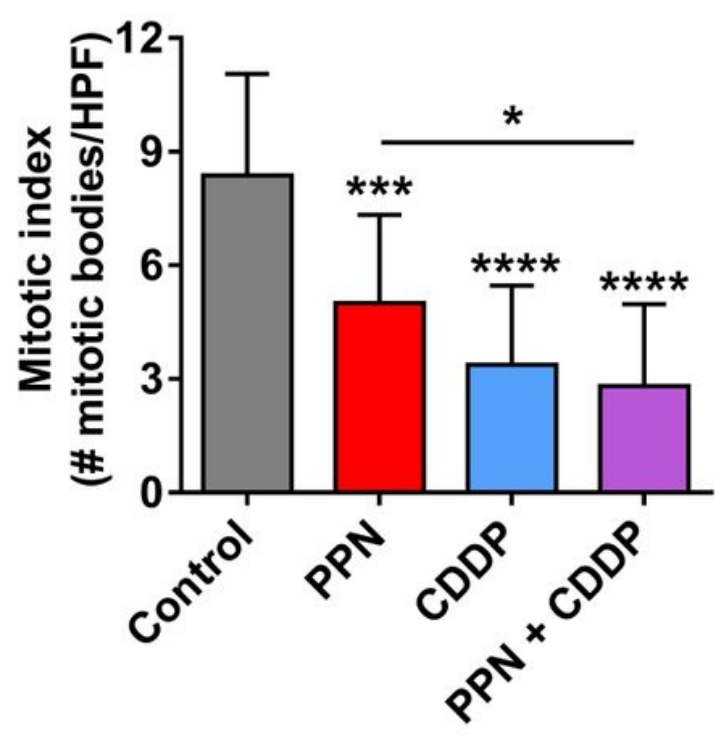

C

\section{Adjusted tumor necrotic rate (\%)}

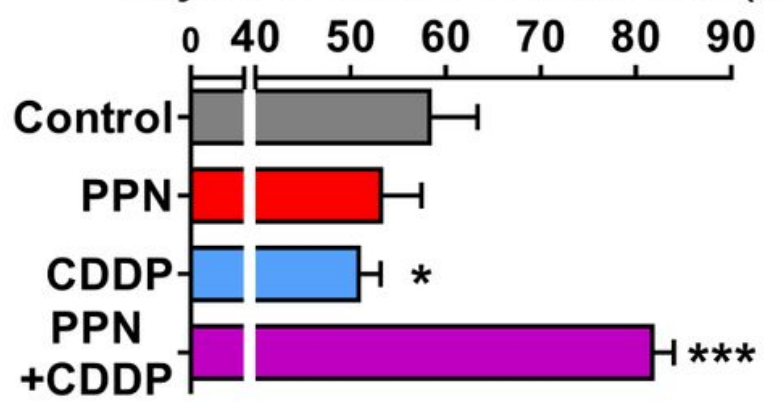

b
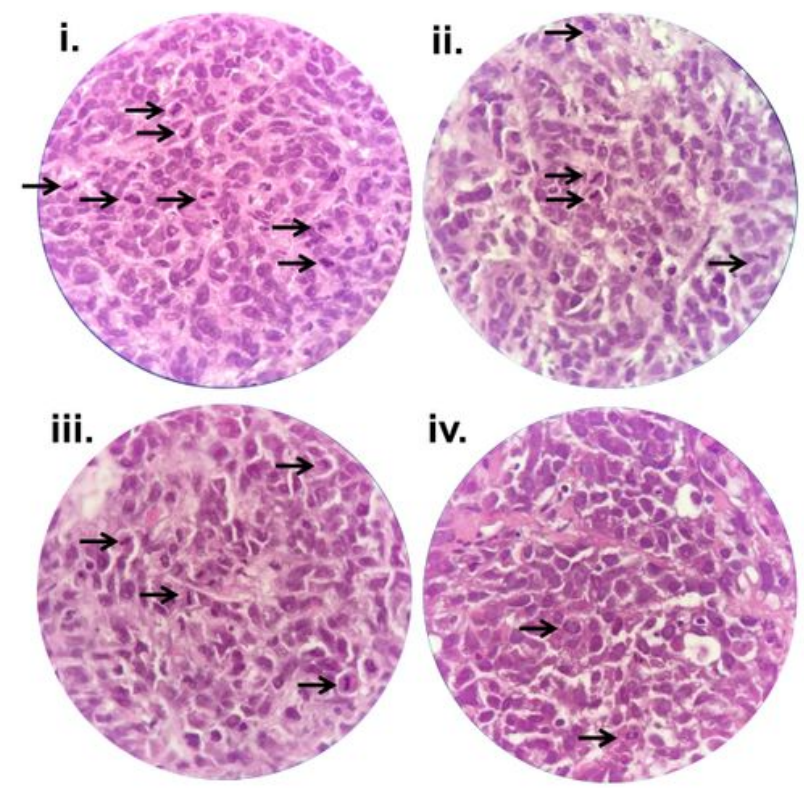

iv.

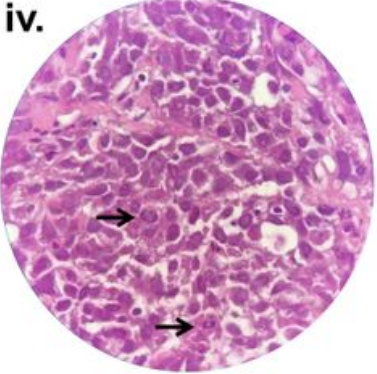

d
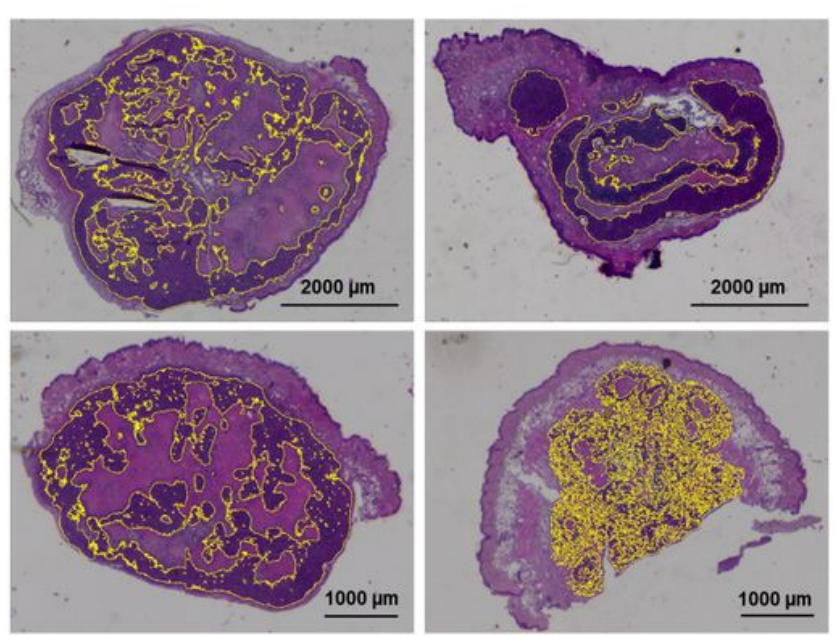

\section{Figure 5}

Histopathological assessment of mitotic index and necrosis in osteosarcoma tumors after propranolol treatment, alone or in combination with cisplatin. (a) Antimitotic activity of PPN in combination to metronomic CDDP on OSA xenografts. Quantification of mitotic index in OSA samples from different experimental groups was expressed as number of mitotic bodies per HPF. (b) Representative H\&E stained tumor slides belonging to animals treated with i) vehicle, ii) PPN, iii) CDDP or iv) PPN plus CDDP. Arrows show OSA cell mitotic bodies. X400 magnification. (c) \% of tumor necrosis in OSA samples was adjusted to tumor volume variation as a response to specific treatments, and was expressed as adjusted tumor necrotic rates. (d) Representative digitally-stitched images of complete tumor sections from vehicle (upper left), PPN (upper right), CDDP (lower left) and PPN plus CDDP (lower right) treatment groups. Yellow line in tumor slides mark highly basophilic tissue regions belonging to viable malignant tissue. Scale bars $=1000$ or $2000 \mu \mathrm{m}$. Data are presented as mean \pm SEM. ${ }^{*} p<0.05,{ }^{* \star *} p<0.001,{ }^{* \star * *} p<0.0001$. ANOVA followed by Tukey's test. 


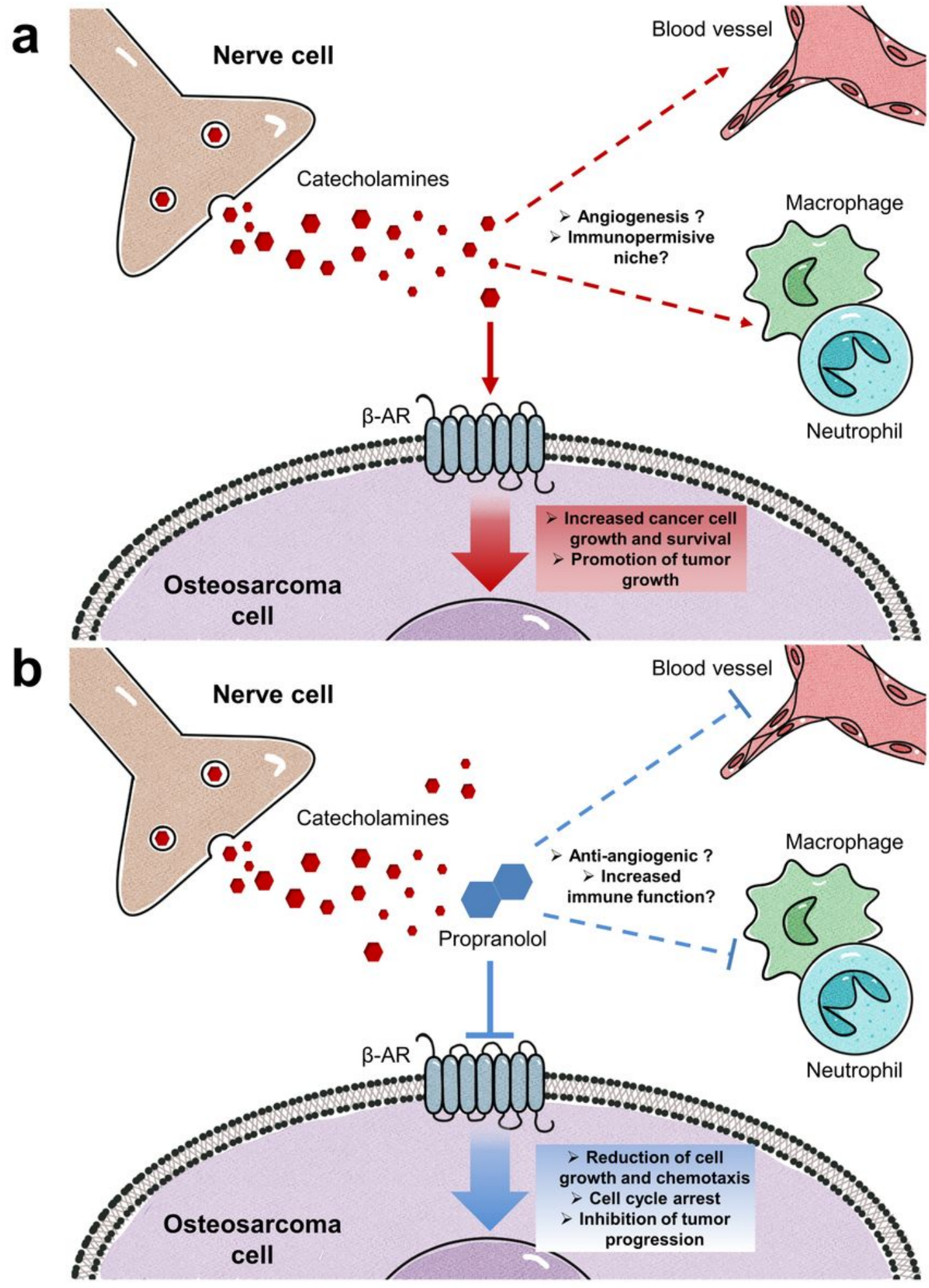

\section{Figure 6}

Potential antitumor activity and therapeutic benefits of repurposed $\beta$-blocker propranolol in osteosarcoma. (a). $\beta$-AR signaling is involved in cancer progression and is upregulated in osteosarcoma. Its activation by catecholamines in tumor cells, and probably in other stromal players such as vascular and immune cells, promotes proliferation and osteosarcoma growth. (b) $\beta$-AR antagonization using propranolol blocks protumoral effects of catecholamines, and inhibits osteosarcoma growth, especially 
in addition to chemotherapy. Direct activity of propranolol in cancer cells, as well as other tumor stromamediated mechanisms of action, should be further explored in osteosarcoma.

\section{Supplementary Files}

This is a list of supplementary files associated with this preprint. Click to download.

- Additionalfile1.Supplementarymethods.docx

- Additionalfile2.FigureS1.tif 